\title{
Caliceal Fluid Temperature During High-Power Holmium Laser Lithotripsy in an In Vivo Porcine Model
}

\author{
Ali H. Aldoukhi, MD, MS, Timothy L. Hall, PhD, ${ }^{2}$ Khurshid R. Ghani, MD, MS, ${ }^{1}$ Adam D. Maxwell, PhD, \\ Brian MacConaghy, and William W. Roberts, $\mathrm{MD}^{1,2}$
}

\begin{abstract}
Introduction: With increasing use of high-power laser settings for lithotripsy, the potential exists to induce thermal tissue damage. In vitro studies have demonstrated that temperature elevation sufficient to cause thermal tissue damage can occur with certain laser and irrigation settings. The objective of this pilot study was to measure caliceal fluid temperature during high-power laser lithotripsy in an in vivo porcine model.

Methods: Four female pigs ( $30-35 \mathrm{~kg}$ ) were placed under general anesthesia and positioned supine. Retrograde ureteroscopy with entry into upper or middle calices was performed. Thermocouples were placed into the calix by open exposure and puncture of the kidney or retrograde alongside the ureteroscope. A $242 \mu \mathrm{m}$ laser fiber was positioned in the center of the calix and activated $(0.5 \mathrm{~J}, 80 \mathrm{~Hz}, 40 \mathrm{~W})$ for 60 seconds with high, medium, or no irrigation delivered in each trial. Finite element simulations of laser-induced heating in a renal calix were also performed.

Results: Peak temperatures of $84.8^{\circ} \mathrm{C}, 63.9^{\circ} \mathrm{C}$, and $43.6^{\circ} \mathrm{C}$ were recorded for no, medium, and high irrigation, respectively. Mean time to reach threshold of thermal injury ( $t_{43}$ of 120 minutes) was 12.7 and 17.8 seconds for no and medium irrigation. Thermal damage thresholds were not reached in high-irrigation trials. Numerical simulations revealed similar results with peak spatial average fluid temperatures of $>100^{\circ} \mathrm{C}, 58.5^{\circ} \mathrm{C}$, and $37.5^{\circ} \mathrm{C}$ during 60 seconds of laser activation for $0.1,15$, and $40 \mathrm{~mL} /$ minute irrigation, respectively.

Conclusions: High-power holmium laser settings $(40 \mathrm{~W})$ can induce potentially injurious temperatures in the porcine in vivo model, particularly with slower irrigation rates. Characterization of thermal dose across a broader range of laser parameter settings is underway to map out the thermal safety envelope.
\end{abstract}

Keywords: holmium laser, temperature, lithotripsy, ureteroscopy, in vivo

\section{Introduction}

$\mathbf{T}$ HE INTRODUCTION OF high-power holmium laser systems $(100-120 \mathrm{~W})$ has expanded available parameter settings and ushered in a new era of ureteroscopic stone treatment. Laser lithotripsy can now be applied with higher frequency, enabling strategies of "dusting" and "popdusting" where stone or stone fragments are eroded to submillimeter particles (common parameters: $0.2-0.5 \mathrm{~J}$ pulse energy; $50-80 \mathrm{~Hz}$ frequency). ${ }^{1}$ These new "dusting" strategies are effective adjuncts to traditional fragmentation laser lithotripsy and have been shown to shorten operative time. ${ }^{2}$ In many cases, a combination of fragmentation and dusting modes of operation is necessary to treat particularly large or "hard" stones. A survey in 2015 revealed that $67 \%$ of endourologists commonly used some form of stone "dusting" technique. ${ }^{3}$
These laser strategies use increased power settings (up to $40 \mathrm{~W}$ ), frequently applied as 5 to 10 second bursts of laser activation, and often with bursts applied in rapid succession. With these settings, the potential exists to overheat the caliceal fluid as demonstrated in recent bench studies. ${ }^{4-7}$ Temperatures in bench models were seen to reach $70^{\circ} \mathrm{C}$ after 60 seconds of firing at $40 \mathrm{~W}$ laser settings, ${ }^{3}$ generating a thermal dose that would be sufficient to damage adjacent urothelium, renal parenchyma, and/or ureter. This study was conducted to determine whether similar temperature elevation also occur in the in vivo setting. The specific objective was to measure caliceal fluid temperature during high-power laser lithotripsy in an in vivo porcine model.

\section{Methods}

After approval from the Institutional Animal Care and Use Committee, four female pigs $(30-35 \mathrm{~kg})$ were anesthetized,

Departments of ${ }^{1}$ Urology and ${ }^{2}$ Biomedical Engineering, University of Michigan, Ann Arbor, Michigan.

${ }^{3}$ Department of Urology, University of Washington School of Medicine, Seattle, Washington.

${ }^{4}$ Applied Physics Laboratory, University of Washington, Seattle, Washington.

A video demonstrating this technique is available online at www.liebertpub.com/end 
intubated, and positioned supine (Fig. 1A). Five kidneys were exposed with a modified subcostal incision without entering the peritoneum (Fig. 1B). A flexible cystoscope (CYF-3; Olympus) was used to insert a guidewire through the ureter into the renal pelvis. Retrograde ureteroscopy with entry into upper or interpolar renal calices was performed with a flexible ureteroscope (DUR-8 Gyrus ACMI). With visual (ureteroscope) and ultrasound guidance (GE P6 ultrasound scanner with $11 \mathrm{~L}$ probe), a type $\mathrm{T}$ needle thermocouple (Physitemp) was inserted through the exposed kidney and into the targeted calix. In two kidneys, rather than surgical exposure, a wire thermocouple (Omega) was attached to the ureteroscope shaft with its tip $2 \mathrm{~mm}$ from the distal end and inserted retrograde into the renal collecting system with the ureteroscope (Fig. 1C). Data from one kidney were excluded as experimental protocol was not followed because of early termination of laser energy delivery when temperature exceeded $100^{\circ} \mathrm{C}$.

Twenty trials were performed in seven kidneys with high (6), medium (7), and no (7) irrigation. High irrigation was established with saline contained in a pressure bag inflated to $150 \mathrm{mmHg}$ and placed at $100 \mathrm{~cm}$ height, equivalent to $304 \mathrm{~cm}$ of gravity irrigation. Medium irrigation consisted of a saline bag at $100 \mathrm{~cm}$ height. The irrigation rate measured with a $242 \mu \mathrm{m}$ laser fiber in the working channel and no outflow resistance was 36 to 38 and 14 to $15 \mathrm{~mL} /$ minute for high and medium irrigation, respectively.

During each trial, a $242 \mu \mathrm{m}$ laser fiber (Flexiva; Boston Scientific) was introduced through the working channel of the ureteroscope with laser fiber tip positioned in the center of the calix away from the thermocouple and caliceal wall. All trials were conducted with laser energy output of $40 \mathrm{~W}(0.5 \mathrm{~J}$, $80 \mathrm{~Hz}$, short pulse) using a $120 \mathrm{~W}$ holmium laser system (pulse 120; Lumenis). Each trial was conducted for 100 seconds with continuous temperature measurement. Baseline temperature was recorded for the first 10 seconds (time 0-10 seconds) after which the laser was activated for 60 seconds (time 11-70 seconds). Temperature was then recorded for an additional 30 seconds (time 71-100 seconds).
One kidney was harvested after three trials at high, medium, and no irrigation. The kidney was bivalved and sectioned transversely through the calix where the laser had been activated to assess gross pathologic changes.

Thermal dose for each trial was calculated based on Sapareto and Dewey $t_{43}$ equivalence calculations for each trial. ${ }^{8}$ Using this concept, the thermal dose of any temperature curve can be converted to an equivalent time ( $t_{43}$ minutes) at a stable temperature (conventionally considered $43^{\circ} \mathrm{C}$ ). Sapareto and Dewey found that a temperature of $43^{\circ} \mathrm{C}$ for 120 to 240 minutes produced thermal injury in various tissues. For the purpose of this evaluation, we consider $t_{43}=120$ minutes to be the threshold for thermal tissue injury.

Finite element simulations were performed for high, medium, and no irrigation in COMSOL Multiphysics (COMSOL, Inc., Burlington, MA). Simulation geometry mimicking the calix consisted of an axisymmetric $6 \mathrm{~mm}$ diameter $50 \mathrm{~mm}$ long tube terminated in a hemispherical end on one side and open at the opposing end. Surrounding this lumen was a 1-mm thick wall of tissue with thermal and mechanical properties of the ureteral wall, which was further surrounded externally by tissue with properties of the kidney parenchyma. The simulation used a bioheat transfer model ${ }^{9}$ to capture the physics of laser heat generation, diffusion, and convection, as well as blood perfusion and fluid flow because of irrigation. A steady irrigation of 40,15 , or $0.1 \mathrm{~mL} /$ minute (simulating high, medium, and no irrigation in the in vivo trials) was emitted from an irrigation port placed at the radial center of the fluid space, $20 \mathrm{~mm}$ from the end. Heat was generated as a $0.5 \mathrm{~mm} 40 \mathrm{~W}$ spherical source $15 \mathrm{~mm}$ from the end of the calix. To simulate the temporal heat profile in vivo, fluid irrigation was introduced for 20 seconds, after which the laser heating was generated for 60 seconds, then temperature was recorded for 20 seconds more. Fluid average temperature and thermal dose were calculated at each point in the field.

Data are presented as mean and standard deviation. Statistical analyses were performed using Microsoft Excel (Redmond, WA).
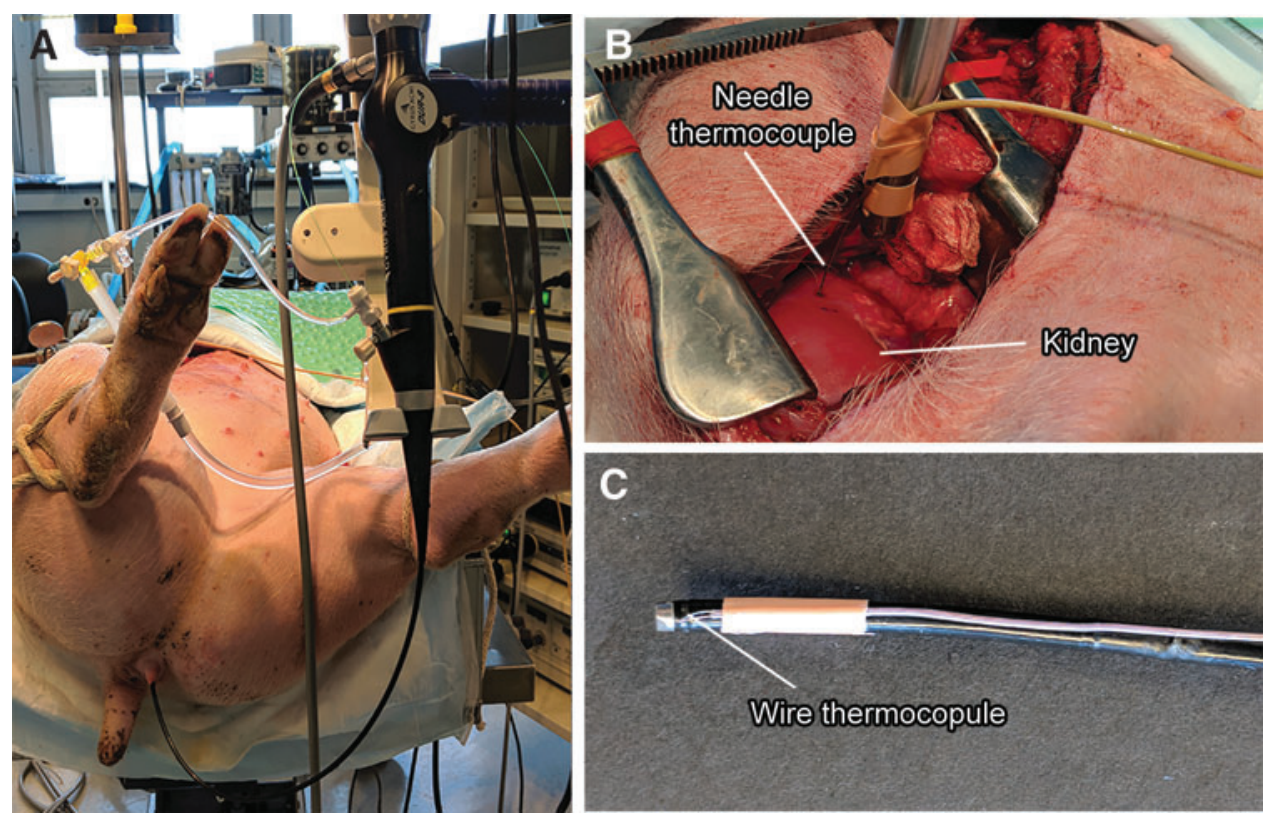

FIG. 1. Experimental setup showing (A) the pig positioned supine on the table with ureteroscope inserted to renal calix, (B) modified subcostal incision showing the exposed kidney and needle thermocouple, and (C) wire thermocouple secured to the ureteroscope. 
FIG. 2. Temperature recordings for all trials with no irrigation. Laser energy was applied starting at 10 seconds and ending at 70 seconds.

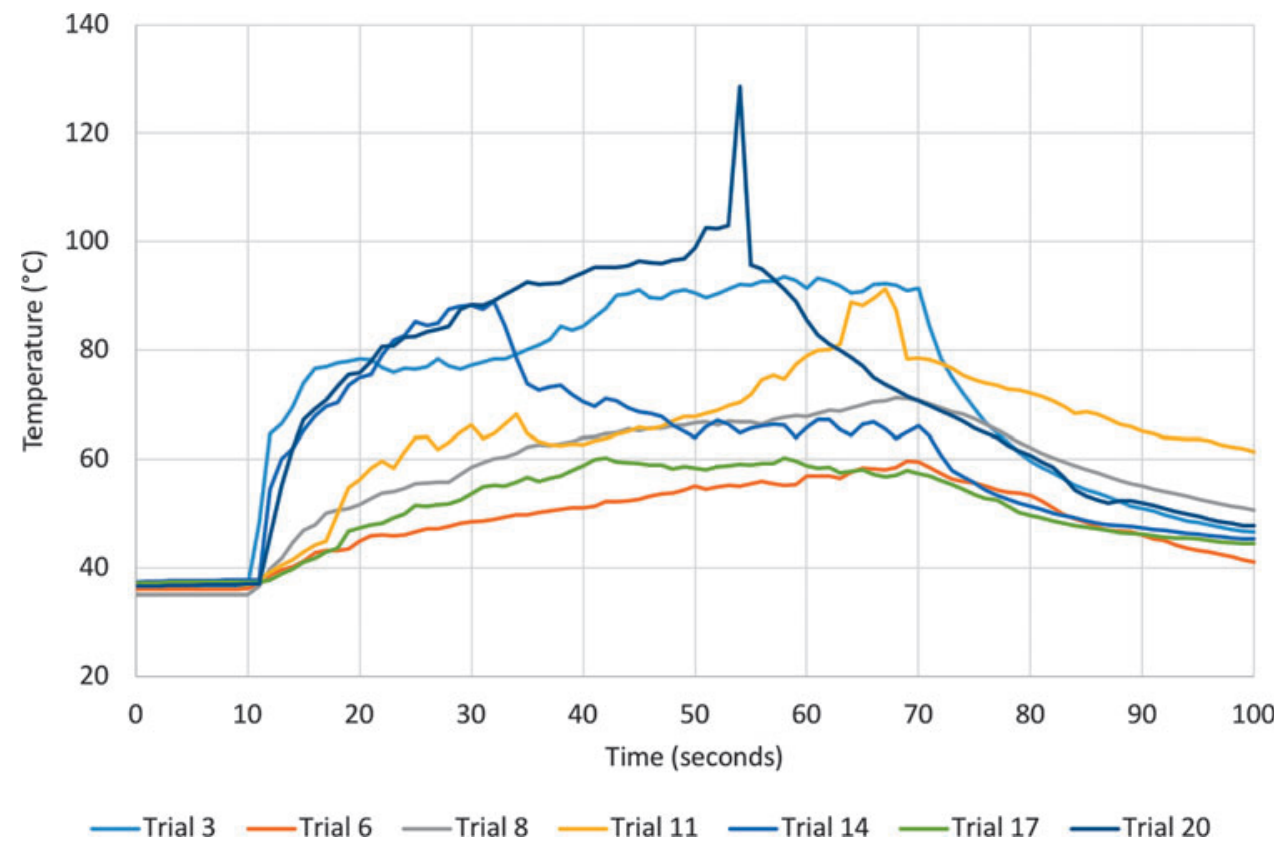

\section{Results}

Caliceal fluid temperature increased in all trials during laser activation (Figs. 2 and 3 and Supplementary Fig. S1; Supplementary Data are available online at www .liebertpub.com/end) with the highest temperature, $128.5^{\circ} \mathrm{C}$, recorded during a trial with no irrigation. Figure 4 shows mean temperature change from baseline for each irrigation rate. After only 10 seconds of laser activation, caliceal fluid temperature reached $61.4^{\circ} \mathrm{C}, 50.1{ }^{\circ} \mathrm{C}$, and $39.2^{\circ} \mathrm{C}$, corresponding to an average temperature increase of $25.4^{\circ} \mathrm{C}$ for no irrigation, $16.9^{\circ} \mathrm{C}$ for medium irrigation, and $10.6^{\circ} \mathrm{C}$ for high irrigation.
The thermal threshold of $t_{43}=120$ minutes was exceeded for all trials with no irrigation and six of seven with medium irrigation (Table 1). The mean time to reach the threshold of thermal injury was 12.7 seconds (range 2-37 seconds) for no irrigation and 17.8 seconds (range 5-30 seconds) for medium irrigation. In one trial with medium irrigation and all trials with high irrigation, thermal damage thresholds were not exceeded during 60 seconds of laser activation.

Numerical simulations recorded spatial average fluid temperatures as high as $>100^{\circ} \mathrm{C}, 58.5^{\circ} \mathrm{C}$, and $37.5^{\circ} \mathrm{C}$ within 60 seconds of laser power for $0.1,15$, and $40 \mathrm{~mL} / \mathrm{second}$ irrigation, respectively. Simulations did not show fluid mixing at the $0.1 \mathrm{~mL} / \mathrm{minute}$ case, thus concentrating heat around
FIG. 3. Temperature recording for all trials with medium irrigation. Laser energy was applied starting at 10 seconds and ending at 70 seconds.

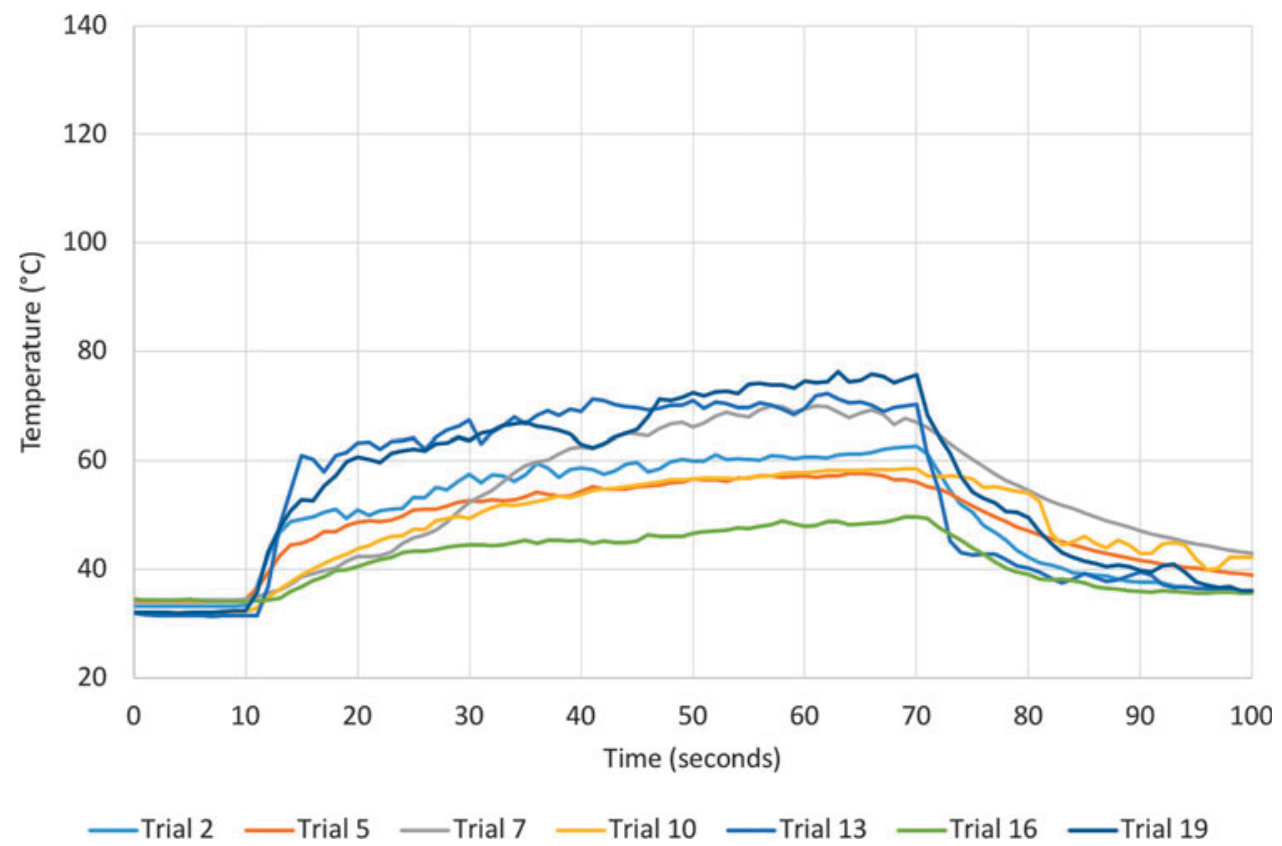




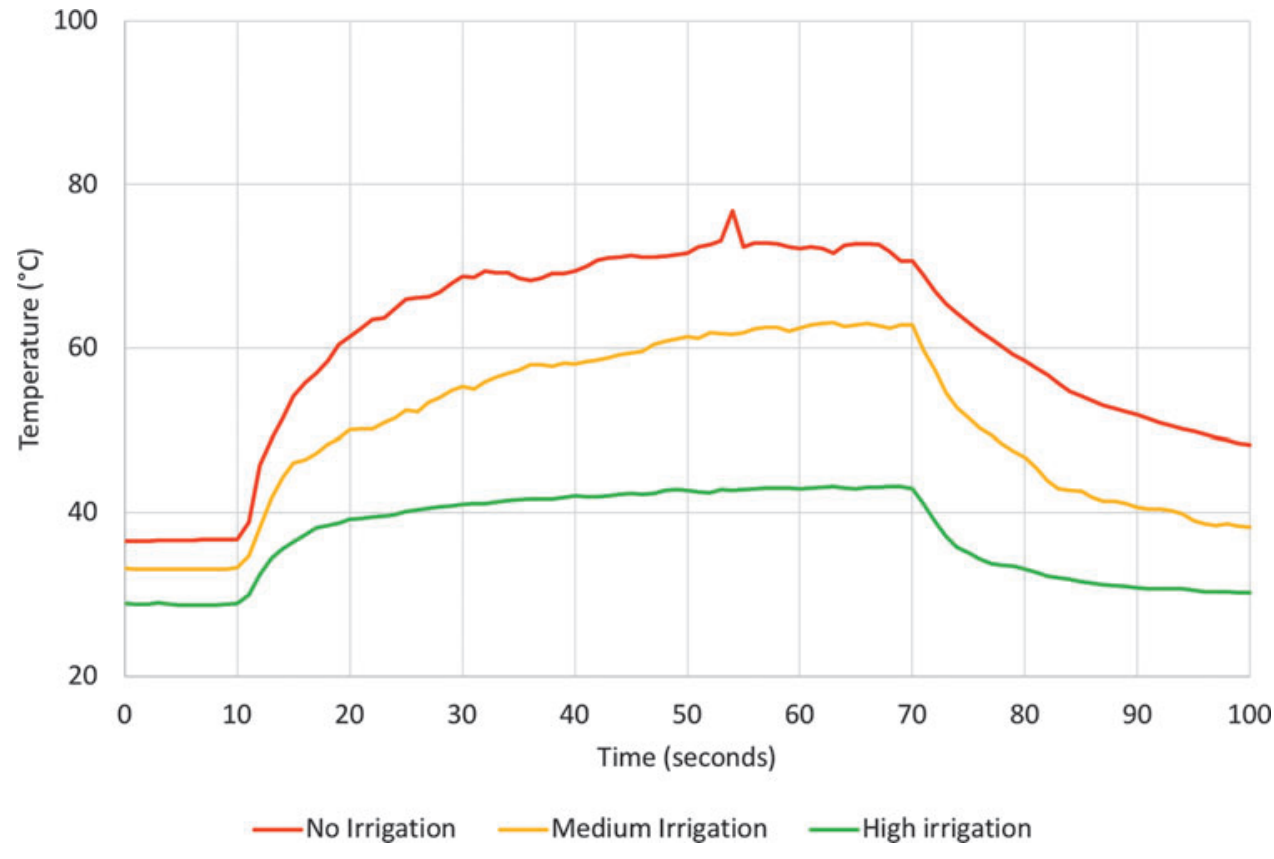

FIG. 4. Mean temperature with each irrigation rate. Laser energy was applied starting at 10 seconds and ending at 70 seconds. the laser fiber tip. However, the fluid temperature was relatively uniform throughout the calix when irrigation was introduced. Time to onset of thermal injury based on the thermal dose anywhere in the tissue was calculated at 4.1 and 4.9 seconds for 0.1 and $15 \mathrm{~mL} /$ minute irrigation.

Gross pathologic tissue coagulation and injury were apparent in the one kidney harvested for gross assessment (Fig. 5). Charred (black) tissue is seen at the urothelial surface in contact with the collecting system. This is surrounded by a white zone of coagulation surrounding the margin of the calix. A concentric hyperemic (red) zone extends beyond the coagulation zone further into paren- chyma. Tissue temperatures consistent with these findings were also demonstrated by numerical simulation. In the no-irrigation state, the thermal threshold boundary extended beyond the calix, urothelium, and $3 \mathrm{~mm}$ into the adjacent parenchyma over a length of $\sim 14 \mathrm{~mm}$ (Supplementary Fig. S2 and Supplementary Video S1). In the medium irrigation state, injury was limited to a thin area $(<1 \mathrm{~mm}$ depth $)$ within the wall of the calix (Fig. 6 and Supplementary Video $\mathrm{S} 2$ ). Total volumetric injury in simulations was up to $2.3 \mathrm{~cm}^{3}$ $\left(0.1 \mathrm{~mL} /\right.$ minute irrigation) and $0.28 \mathrm{~cm}^{3}(15 \mathrm{~mL} /$ minute irrigation). No injury was produced anywhere in the field with $40 \mathrm{~mL} /$ minute irrigation.

Table 1. Data Recorded During All Trials with $T_{43}$ Equivalent Calculation

\begin{tabular}{|c|c|c|c|c|c|c|}
\hline Trial & $\begin{array}{l}\text { Irrigation } \\
\text { rate }\end{array}$ & $\begin{array}{c}\text { Thermocouple } \\
\text { type }\end{array}$ & $\begin{array}{c}\text { Temperature } \\
\text { after } 10 \text { seconds } \\
\text { of laser application }\left({ }^{\circ} \mathrm{C}\right)\end{array}$ & $\begin{array}{l}\text { Time to } \mathrm{t}_{43} 120 \text { minutes } \\
\text { equivalent (seconds) }\end{array}$ & $\begin{array}{c}\text { Peak } \\
\text { temperature }\left({ }^{\circ} \mathrm{C}\right)\end{array}$ & $\begin{array}{l}\mathrm{t}_{43} \text { Equivalent } \\
\text { minutes }\end{array}$ \\
\hline 3 & No & Needle & 78.4 & 2 & 93.5 & $>10^{13}$ \\
\hline 6 & No & Needle & 45.0 & 37 & 59.7 & $>10^{3}$ \\
\hline 8 & No & Needle & 51.7 & 14 & 71.3 & $>10^{6}$ \\
\hline 11 & No & Needle & 56.3 & 10 & 91.2 & $>10^{11}$ \\
\hline 14 & No & Needle & 75.0 & 3 & 89.0 & $>10^{11}$ \\
\hline 17 & No & Wire & 47.4 & 21 & 60.3 & $>10^{3}$ \\
\hline 20 & No & Wire & 75.8 & 2 & 128.5 & $>10^{22}$ \\
\hline 2 & Medium & Needle & 51.1 & 17 & 62.7 & $>10^{4}$ \\
\hline 5 & Medium & Needle & 48.7 & 25 & 57.7 & $>10^{3}$ \\
\hline 7 & Medium & Needle & 42.4 & 23 & 70.0 & $>10^{6}$ \\
\hline 10 & Medium & Needle & 43.8 & 30 & 58.6 & $>10^{3}$ \\
\hline 13 & Medium & Needle & 63.4 & 5 & 72.3 & $>10^{6}$ \\
\hline 16 & Medium & Wire & 40.6 & NA & 49.7 & 19.6 \\
\hline 19 & Medium & Wire & 60.8 & 7 & 76.3 & $>10^{8}$ \\
\hline 1 & High & Needle & 41.1 & N/A & 47.6 & 6.2 \\
\hline 4 & High & Needle & 42.3 & N/A & 50.1 & 47.5 \\
\hline 9 & High & Needle & 37.4 & N/A & 43.4 & $<0.1$ \\
\hline 12 & High & Needle & 36.8 & N/A & 37.9 & $<0.1$ \\
\hline 15 & High & Wire & 38.9 & N/A & 39.8 & $<0.1$ \\
\hline 18 & High & Wire & 38.8 & N/A & 42.7 & 0.1 \\
\hline
\end{tabular}




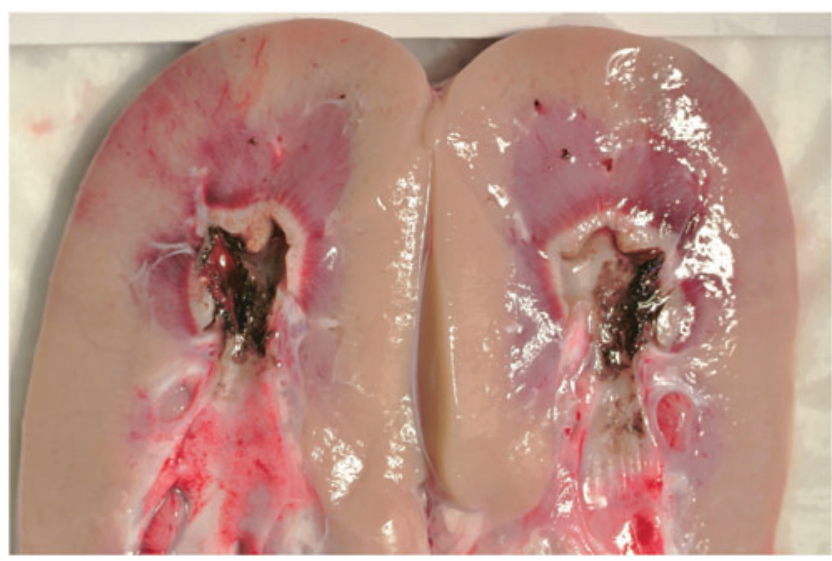

FIG. 5. Gross image of a bivalved kidney showing charred urothelium and thermal injury after three (60 seconds) trials at high, medium, and no irrigation.

\section{Discussion}

The evolution of laser lithotripsy has seen the recent introduction of high-power laser systems, borrowed from the realm of tissue ablation and prostate enucleation. Although the expanded parameter space produces many benefitsbetter stone comminution, smaller fragment size, and faster treatment times - the assessment of thermal safety in laser lithotripsy has lagged behind the clinical introduction of these techniques. The rationale for this study was to explore a limited portion of the parameter space at the upper end of the power range to determine whether toxic thermal dose can also be generated in the in vivo setting.

Recent in vitro bench studies assessing temperature change with holmium laser activation have concluded that highpower low-irrigation settings can lead to temperature increase sufficient to cause tissue injury. ${ }^{4-7}$ Our group previously has shown in an in vitro bench model that temperatures can reach $50.7^{\circ} \mathrm{C}, 45.2^{\circ} \mathrm{C}$, and $37.8^{\circ} \mathrm{C}$ after only 10 seconds of laser activation with no, medium, and high irrigation, respectively. ${ }^{4}$ It was thought that vascular perfusion of the kidneys in the in vivo model would act as a heat sink and dampen the temperature elevations that had been measured in
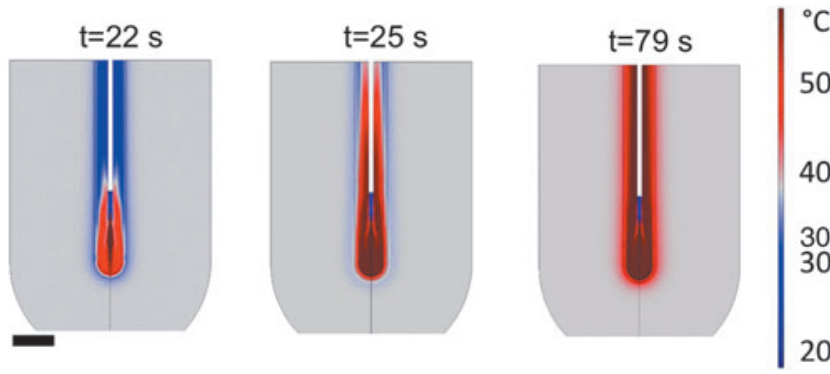

FIG. 6. Numerical simulation of temperature distribution after 2, 5, and 59 seconds of laser activation with $15 \mathrm{~mL} /$ minute irrigation. Room temperature irrigation fluid was introduced through a simulated ureteroscope (white tube) and started 20 seconds before laser activation. Simulated laser heating is generated by a small source $5 \mathrm{~mm}$ below the simulated ureteroscope, causing temperature elevation of the fluid and surrounding tissue. The scale bar is $1 \mathrm{~cm}$. the in vitro bench studies. ${ }^{410}$ Surprisingly, this effect was not observed and in fact, peak temperature in the no irrigation scenario was greater in the in vivo model $\left(84.8^{\circ} \mathrm{C}\right)$ than in the in vitro model $\left(69.8^{\circ} \mathrm{C}\right)$. This may be because of (1) less heat conduction away from the collecting system into the renal tissue and surrounding retroperitoneal structures in vivo compared with the relative ease of conduction from the test tube to the surrounding water bath in vitro and (2) the short time course of energy delivery during laser lithotripsy that likely overwhelms the heat-sink capacity of vascular perfusion.

Numerical simulations of laser-induced heating showed similar temperature elevations with irrigation, although the case with $0.1 \mathrm{~mL} /$ minute irrigation produced significantly higher temperatures than those measured in vivo. This discrepancy is likely caused by evaporation/condensation processes, as well as fluid motion and mixing that occur during laser firing and redistribute heat, but are not modeled here.

The thermal doses observed in prior in vitro studies, numerical simulations, and the in vivo results described here are capable of damage to the urothelium, kidney, and ureter potentially resulting in scarring, obstruction to the ureter or collecting system, and loss of renal function. Although tissue changes associated with thermal injury may not be apparent to urologists at the time of laser lithotripsy and likely encompass only a small fraction of the total renal tissue, no degree of irreversible thermal parenchymal tissue injury should be considered acceptable. This is particularly important for patients with multiple recurrent stones who may need many lithotripsy procedures over the course of their lifetime.

Although the experimental data presented here and in prior bench studies suggest that increased irrigation rates may be able to partially control temperature elevation, ${ }^{4-7}$ this is often cumbersome or difficult to fully implement in the clinical setting. Furthermore, prevailing endourologic trends have been focused on decreasing intrarenal pressures to reduce infectious and hemorrhagic complications. ${ }^{11-13}$ This can be accomplished by lowering irrigation rates although this would then increase risk of thermal injury. Alternatively, use of an access sheath during ureteroscopy has been shown to reduce intrarenal pressure and improve irrigant flow through the ureteroscope during ureteroscopy. ${ }^{13}$ However, this is not possible in every case and often necessitates a ureteral stent afterward. This provides an opportunity and rationale for small profile active (suction) removal of collecting system fluid to maintain sufficient irrigation rates. In addition, chilled irrigation fluid may be of benefit. Another measure to minimize the effect of thermal injury is intermittent laser activation. Activating the laser for shorts periods would allow temperature to return to baseline. However, the temperature decay curves as seen in Figures 2 to 4 suggest that the needed time between laser bursts may be longer than desirable for efficient treatment. Characterization and control of the "operator dutycycle" of the laser are an important area for future study.

There are several limitations to this study. First, 60 seconds of continuous laser activation is not necessarily realistic with respect to clinical practice. However, it does provide standardization for these experiments and creates a full temperature curve that also provides useful temperature data at earlier time points. This is useful for further developing computerized simulations and can be used to determine time to toxic thermal dose. Second, only one laser power setting, $40 \mathrm{~W}$, was assessed, which does not allow prediction of effects at lower power 
settings. Third, the calix size in which the trials were conducted was not controlled and likely contributed to some of the temperature measurement variability, although this is an expected consequence of in vivo research and clinical therapy. Finally, temperature was recorded using two methods, needle and wire thermocouple. Although one could consider this an unwanted variable in the study, it is representative of ongoing development of experimental technique, and did not appear to produce any systematic bias of the measured thermal curves.

Further research is needed to more completely explore a broader swath of the parameter space. Much of this can be done with simulation and in vitro trials as there was high concordance between the results of the previous in vitro studies and the current in vivo study. Also, investigating the effect of temperature change based on realistic laser on-off activation times, "operator duty-cycle" would help elucidate temperatures and thermal doses expected in actual clinical scenarios. Each of these areas of exploration is necessary to, first, define the thermal "safety envelope" within the parameter space provided by high-power laser lithotripsy systems and, second, develop thermal mitigation tools and techniques that enhance laser lithotripsy efficiency while maintaining thermal safety.

In conclusion, delivery of high-power $(40 \mathrm{~W})$ holmium laser energy can induce potentially injurious temperature elevations in the porcine in vivo model, particularly with lower irrigation rates. Further characterization of thermal dose across a broader range of laser parameter settings is underway to map out the thermal safety envelope. This will provide a baseline from which techniques and tools to control and mitigate thermal effects during holmium laser lithotripsy can be developed.

\section{Acknowledgment}

This work was partially funded by NIH K01 DK104854.

\section{Author Disclosure Statement}

KRG is a consultant for Boston Scientific and Lumenis.

\section{References}

1. Aldoukhi AH, Roberts WW, Hall TL, Ghani KR. Holmium laser lithotripsy in the new stone age: Dust or bust? Front Surg 2017;4:57.

2. Humphreys MR, Shah OD, Monga M, et al. Dusting versus basketing during ureteroscopy-which technique is more efficacious? A prospective multicenter trial from the EDGE Research Consortium. J Urol 2018;199:1272-1276.
3. Dauw CA, Simeon L, Alruwaily AF, et al. Contemporary practice patterns of flexible ureteroscopy for treating renal stones: Results of a worldwide survey. J Endourol 2015;29: 1221-1230.

4. Aldoukhi AH, Ghani KR, Hall TL, Roberts WW. Thermal response to high-power holmium laser lithotripsy. J Endourol 2017;31:1308-1312.

5. Wollin DA, Carlos EC, Tom WR, Simmons WN, Preminger GM, Lipkin ME. Effect of laser settings and irrigation rates on ureteral temperature during holmium laser lithotripsy, an in vitro model. J Endourol 2018;32:59-63.

6. Buttice S, Sener TE, Proietti S, et al. Temperature changes inside the kidney: What happens during holmium:yttriumaluminium-garnet laser usage? J Endourol 2016;30:574-579.

7. Molina WR, Silva IN, Donalisio da Silva R, Gustafson D, Sehrt D, Kim FJ. Influence of saline on temperature profile of laser lithotripsy activation. J Endourol 2015;29:235-239.

8. Sapareto SA, Dewey WC. Thermal dose determination in cancer therapy. Int J Rad Oncol Biol Phys 1984;10:787-800.

9. Pennes HH. Analysis of tissue and arterial blood temperatures in the resting human forearm. J Appl Physiol 1948;1: 93-122.

10. Goldberg SN, Hahn PF, Tanabe KK, et al. Percutaneous radiofrequency tissue ablation: Does perfusion-mediated tissue cooling limit coagulation necrosis? J Vasc Interv Rad 1998;9:101-111.

11. Auge BK, Pietrow PK, Lallas CD, Raj GV, Santa-Cruz RW, Preminger GM. Ureteral access sheath provides protection against elevated renal pressures during routine flexible ureteroscopic stone manipulation. J Endourol 2004; 18:33-36.

12. Jung H, Norby B, Frimodt-Moller PC, Osther PJ. Endoluminal isoproterenol irrigation decreases renal pelvic pressure during flexible ureterorenoscopy: A clinical randomized, controlled study. Eur Urol 2008;54:1404-1413.

13. Rehman J, Monga M, Landman J, et al. Characterization of intrapelvic pressure during ureteropyeloscopy with ureteral access sheaths. Urology 2003;61:713-718.

Address correspondence to: William W. Roberts, MD Department of Urology University of Michigan Taubman Center 3879 1500 E Medical Center Dr. Ann Arbor, MI 48109

E-mail: willrobe@med.umich.edu 